\title{
ANALYTICAL APPROXIMATIONS OF LONG-EXPOSURE POINT SPREAD FUNCTIONS AND THEIR USE
}

\author{
O.Bendinelli ${ }^{1}$, G.Parmeggiani ${ }^{2}$, F.Zavatti ${ }^{1}$ \\ 1 Dipartimento di Astronomia, Universita' di Bologna \\ 2 Osservatorio Astronomico di Bologna
}

\begin{abstract}
The observed light distribution in long exposure star images (PSF) may be fitted equally well by a variety of models. But dealing with undersampled star images, only the use of the multi-Gaussian model allows the correct model parameters estimation, taking into account integration on pixel surface, image off-centering and background behaviour. It is also shown that the convolution of a spherical source with the multi-Gaussian and Moftat's models gives in practice the same result.
\end{abstract}

\section{Introduction}

It is known that seeing theory does not give a simple description for the light distribution in long exposure star images, as done for short exposure images by Fried (1966). Nevertheless, its behaviour is well known empirically as it appears from the pioneering works by de Vaucouleurs $(1948,1958)$ and the more recent ones by King (1971) and Kormendy (1973). The PSF results nearly Gaussian at the centre, with extended wings following approximatively first an exponential and after an inverse power law fading in the background. The energy encircled in these zones clearly depends on the sky condition during the observations, typical values being of the order of 60,30 and 10 per cent of the total. It must be stressed that the external zone, containing a small fraction of the total light spreaded over a very large region is generally negligible in observations reduction, excepting particular cases as detection of a faint star imbedded in the halo of a much brighter one or determination of the surface brightness of a distant galaxy with a very bright nucleus (see Schweizer, 1979 and Djorgovski, 1984). In any case, the extreme wings of the PSF are too poorly known, the only information available being the 3 values of the power $-2.0,-1.5$ and -1.7 , due respectively to King (1971), Kormendy (1973) and C'apaccioli and de Vaucouleurs (1979).

\section{PSF empirical models}

Among the variety of empirical smooth fitting functions proposed to represent long-exposures PSFs, the more widely employed are the Moffat (1969) distribution, a central truncated Gaussian overlapping an exponential wing (King, 1971), a generalization of the Lorentzian (Franz, 1973) , a mixture of Gaussians (Brown, 1974) and the sum of a Gaussian and an exponential convolved with the same Gaussian (Lauer, 1985). All these approximations give good fits of oversampled star images, i.e. those characterized by a scale length larger than 


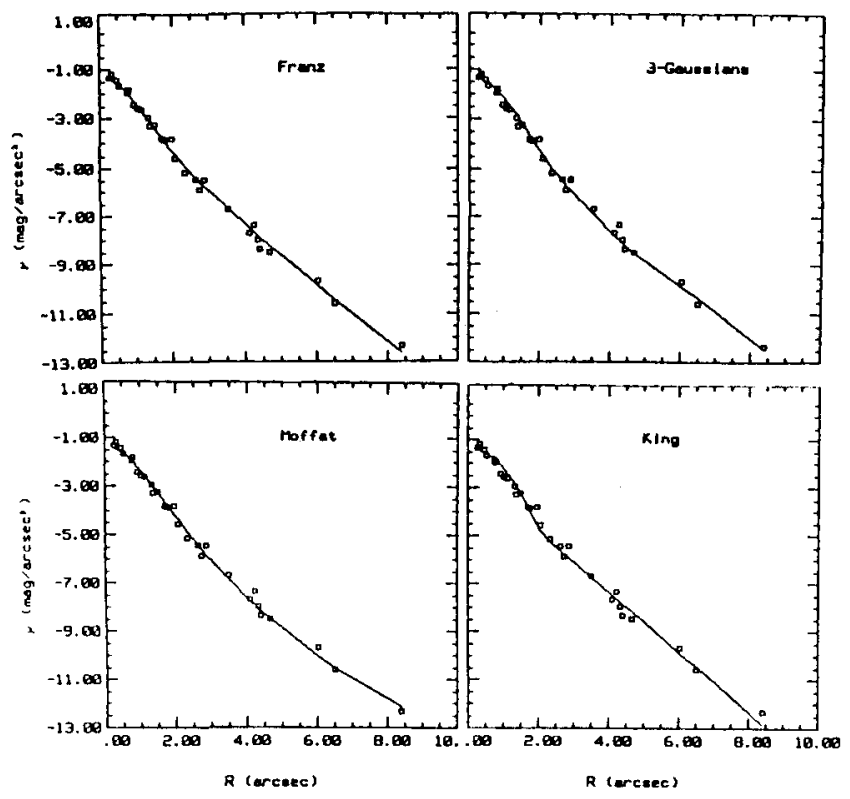

Figure 1: Fitting of King's (1971) star profile by different models (solid line)

the pixel size for CCCD observations or the microdensitometer spot for photografic plates. This is shown in Fig.1, where the composite profile by King (1971) has been fitted by four of the above models.

But a serious problem arises in PSF determination from undersampled images (i.e., those with seeing scale length smaller than pixel or spot size), since neither the integration on the pixel surface nor the off-centering of the image centre from the central pixel (i.e., that of local maximum intensity) can be neglected. The reason of this resides in the analytical properties of models which must be pointed out. First, none of them is linear in the parameters, the worse being from this point of view that by Lauer, in which the convolution of the Gaussian and the Exponential is not expressible in finite terms, and that by Franz where the identifiability conditions are severely violated. In spite of its simplicity, also the King's model is not useful owing to the discontinuity point. Secondly, excepting the multi-Gaussian model, variables are not separable when the pixel (or the spot) integration is taken into account. So, only Moffat's and Brown's models can be used in practice for more sophisticated applications.

\section{PSF determination from CCD frames}

CCD frames with pixel size greater than the dispersion parameter of the Gaussian seeing do not allow easy determination of the PSF for two main reasons. The first is that the apparent radial brightness profile of a star image is smoother than the true seeing profile, being integrated on the pixel surface, the second that the star image, even with constant background, is skewed, unless its centre coincides with the pixel centre. This means that the accurate knowledge of the seeing may be obtained only by simultaneous estimate of all the parameters (including background ones) of the image model, and taking into account the 
integration limits, which are off-centering dependent. In practice the centering algorithms of Van Altena and Auer (1975) and Chiu (1977) are improved by using the analytical properties of Gaussians and the Newton-Gauss regularized method (NGR) to secure the convergence of the iterative process leading to the parameters estimate. As shown in Bendinelli et al. (1987), the marginal distribution $f_{x}\left(x_{n}\right)$ of the light intensity (i.e., the column pixel sums) may be expressed by

$$
\begin{gathered}
f_{x}\left(x_{n}\right)=0.5 \sum_{i} a_{i}\left\{\operatorname{erf}\left[\left(x_{n}+1-x_{c}\right) / \sigma_{i} \sqrt{2}\right]\right. \\
\left.-\operatorname{erf}\left[\left(x_{n}-1-x_{c}\right) / \sigma_{i} \sqrt{2}\right]\right\}+M b+M\left(x_{n}-x_{c}\right) \Delta_{x} b,
\end{gathered}
$$

which is a suitable form for the application of the NGR method since it is easily differentiable with respect to the $2 \mathrm{~N}+3$ parameters $a_{i}, \sigma_{i}, x_{c}, b, \Delta_{x} b$. A similar equation can evidently be derived for $f_{y}\left(y_{m}\right)$ in rewriting Eq.(1). If both the parameters estimates are in reasonable agreement, the PSF is circularly symmetric even though the apparent star image is elliptical due to background gradients. On the contrary, a more sophisticated seeing model could be taken, such as a sum of bivariate Gaussian distributions with dispersion ellipses rotated with respect to the $\mathrm{x}, \mathrm{y}$ axes (see e.g. (hiu, 1977). If one tries to use Moffat's model, the analogous of Eq.(1) cannot be obtained because the model itself is not integrable in finite terms. As shown in Bendinelli et al. (1988), approximate values of Moffat's shape parameters can be derived by the sequence of integrated luminosities over squares surfaces with $n$ pixels sizes $L\left(r_{n}\right)$, expressed by

$$
L\left(r_{\mathrm{n}}\right)=L_{T}\left[1-\left(1+r^{2} / \alpha^{2}\right)^{1-\beta}\right]+A_{\mathrm{n}} f
$$

which enables us to estimate the parameters by the Newton-Gauss regularized method since differentiable with respect to parameters $\alpha, \beta, f, L_{T}$. As far as the off-centering is concerned, it can be derived, before the search of shape parameters, from the moments of the marginal pixel intensity distributions (see Chiu, 1977). The fits of a star image in the M 31 field (obtained by S.G. Djorgovski at the Kitt Peak 4-m telescope with a TI 800x800 CCD, pixel size of 0.298 arcsec) by the above mentioned methods are shown in Fig.2. It is evident that the brightness profiles computed with both models agree reasonably well with the observed data. The models diverge out of about 4.5 arcsec (i.e. roughly speaking $3 \sigma_{3}$ and $7 \alpha$ ), but it should be stressed that the integrated luminosity of Moffat model from $7 \alpha$ to the infinity, see Eq.(2), is less than one hundreth of the total, so that the true brightness distribution in extreme wings is irrelevant for any reasonable application (see, for instance, the next section).

\section{Convolution of a spherical source}

In a series of papers (see Bendinelli et al., 1986, and references therein) it has been shown that convolution-deconvolution of a spherical source with the PSF, approximated by a sum of weighted Gaussians, can be performed by the monodimensional integral equation

$$
f(r)=\sum_{i=1}^{N}\left(a_{i} / \sigma_{i}^{2}\right) \exp \left(-r^{2} / 2 \sigma_{i}^{2} \times \int_{0}^{+\infty} \exp \left(-\rho^{2} / 2 \sigma_{i}^{2}\right) I_{0}\left(r \rho / \sigma_{i}^{2}\right) \phi(\rho) d \rho,\right.
$$

relating the true brightness distribution $\phi(r)$ and the observed one $f(r)$. Let us assume Moffat's PSF approximation, then convolution is expressed by the double integral equation 


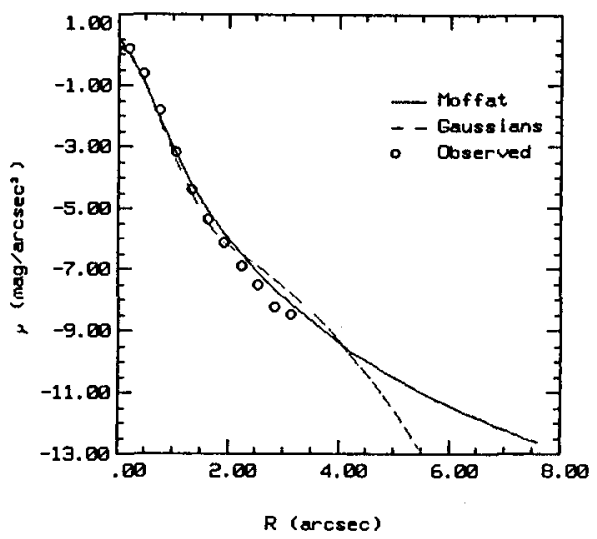

Figure 2: Fits of a star image in M31 field

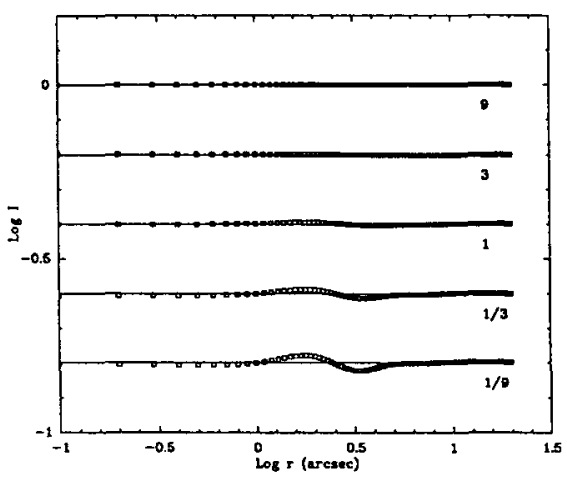

Figure 3: differences in convolved profiles using Moffat and multi-Gaussian approximations

$$
f(r)=\left[2(\beta-1) / \pi \alpha^{2}\right] \int_{0}^{+\infty} \rho F(\rho) \int_{0}^{\pi}\left[1+\left(\rho^{2}+r^{2}-2 \rho r \cos \theta\right) / \alpha^{2}\right]^{-\beta} d \rho d \theta
$$

which requires, to be computed or inverted up to the radial distance where left-hand side term become negligible, about a factor four in time more than Eq.(3). To prove the substantial equivalence of Eqs.(3) and (4), a set of empirical King models has been choosen. They are characterized by the same concentration index $c=2.25$ and $r_{c}$ varying from 9 to 0.11 arcseconds, to simulate distance effect or smaller and smaller intrinsic size. Resulting differences in convolved profiles using Eqs.(3) or (4) are shown in Fig.3.

From the figure some main conclusions can be drawn: i) dealing with large sources $(r>1 ")$ both PSF approximations give practically the same results, ii) for small sources $\left(r<1\right.$ ") local differences of the order of $1 \mathrm{mag} \mathrm{arcsec}^{-2}$ between PSF approximations may cause about $0.1 \mathrm{mag} \operatorname{arcsec}^{-2}$ in convolved profiles, iii) convolution effects become negligible for both PSF approximations at comparable distances from the center, so we must be reasonably confident that the outer profile of distant sources is not an artefact of seeing convolution. 


\section{Concluding remarks}

In conclusion, it seems that parameters of both the multi-Gaussian and Moffat models can be calculated taking into account the finite pixel size, with practically the same accuracy. The use of one model depends on the particular research field. For instance in astrometry the multi-Gaussians is surely preferable, giving also the off-centering, while in extragalactic astronomy the other should be used in order to avoid artefacts of PSF wings on the appearance of distant sources, as discussed for instance by Schweizer (1981) and Djorgovski (1984).

\section{References}

Auer,L.H., Van Altena,W.F.:1978, Astron. J., 83, 531.

Bendinelli,O., Parmeggiani,G., Zavatti,F.: 1986, Astrophys. J., 308, 611.

Bendinelli,O., Parmeggiani,G., Piccioni,A., Zavatti,F.,:1987, Astron. J., 48, 1095.

Bendinelli,O., Parmeggiani,G., Zavatti,F.,:1988, J. Astrophys. Astron., 9, 17.

Brown,G.S.:1974, University of Texas Pub.Astr., No. 11.

Capaccioli,M., de Vaucouleurs,G.: 1979, Astrophys. J. Suppl. Ser., 52, 465.

Chiu,L.T.G.:1977, Astron. J., 82, 842.

de Vaucouleurs,G.: 1948, Ann. Astrophys., 11, 247.

de Vaucouleurs,G.: 1958, Astrophys. J., 128, 465.

Djorgovski,S.:1984, J. Astrophys. Astron., 4, 271.

Franz,O.G.:1973, J. R. Astr. Soc. Can., 67, 81.

Fried,D.L.:1966,J. Opt. Soc. Am.,56,1372.

King,I.R.:1971, Publ. Astron. Soc. Pac., 83, 199.

Kormendy,J.:1973, Astron. J., 78, 255.

Lauer,T.R.:1985, Astrophys. J. Suppl. Ser., 57, 473.

Moffat,A.F.J.:1969, Astron. Astrophys., 3, 455.

Schweizer,F.:1979, Astrophys. J., 233, 23.

Schweizer,F.:1981, Astron. J., 86, 662 .

Van Altena,V.F., Auer,L.H.:1975, in Image Processing Techniques in Astronomy, (Dordrecht:Reidel Pub. Co.), p.411. 